\title{
SOC estimation for Power Lithium-ion Battery Based on AUKF
}

\author{
Enguang Hou ${ }^{a}$, Xin Qiao ${ }^{b}$ and Guangmin Liu ${ }^{c}$ \\ Shandong Provincial Key Laboratory of Automotive Electronic Technology, Institute of Automation, \\ Shandong Academy of Sciences, Jinan 250014, China \\ ahngauto@163.com, bbugqiao@gmail.com, cliugm@keylab.net
}

\begin{abstract}
In order to improve vehicle performance and safety, need to accurately estimate the power lithium battery state of charge (SOC), in allusion to nonlinear characteristic of power lithium-ion battery, presented a method estimation of power lithium-ion battery SOC based on AUKF. Introduced the principle of adaptive estimation based on UKF, and estimated the varying noise statistics, in order to improve the stability of the filter and reduce the estimation error. Simulation results show that the method can convergence even in the case of the initial value is unknown.
\end{abstract}

Keywords: Power lithium-ion battery; Adaptive unscented Kalman filter; SOC; Unscented transform.

\section{Introduction}

Battery state of charge (SOC) is the key issue of electric vehicle battery management system [1]. Accurately estimate battery SOC, the battery can be effectively utilized, in favor of reasonable and effective battery management to improve vehicle performance and safety. Due to the presence of electric vehicle batteries to various internal and external factors during use, which makes an accurate estimate of SOC with great difficulty. At present, the common SOC estimation methods have open circuit voltage, Ah, neural network method and extended Kalman filter (EKF) method [2]. Compared with the previous several methods to calculate the amount of the Kalman filter method is small, easy to implement. However, the Kalman filter method is the noise is assumed to be white noise, and require their statistical properties known. The practical application, noise statistical characteristics may be partially known, approximate known or completely unknown. KF algorithm which based on inaccurate or false noise statistical characteristics of the design will lead to deterioration of filtering performance, even cause filter divergence.

The algorithm has been improved, by experts at home and abroad, and the introduction of UKF. KIM K H[3] presented an adaptive fading extended Kalman filter algorithm for solving stochastic nonlinear system state information partially known estimation of the time, AFEKF algorithm uses a forgetting factor corrected filter gain matrix, and achieved good state estimation results; JWO DJ [4] proposed an adaptive fuzzy strong tracking Kalman filter algorithm is used to solve the system state model cannot accurately estimate the time to get the problem, is constructed a fuzzy logic inference systems by T-S model, the inference system can dynamically adjust the softening factor based on the divergence of the situation, thereby correced the filter gain matrix, and improved the state estimation algorithm performance; State-space equations was established by Mao Hui [5] Based on Thevenin model, accurately SOC estimated in nonlinear conditions by using unscented Kalman filter.

Based on previous studies, proposes an adaptive unscented Kalman filter algorithm, Approximated on nonlinear probability density distribution by UT converted, while adding adaptive estimated principle, to estimated unknown noise, solving the convergence of Kalman filter algorithm, and improve the accuracy of estimates.

\section{Modeling}

The RC equivalent model (as shown in Fig. 1) includes three parts as open-circuit voltage UoC, internal resistances, and equivalent capacitances. The internal resistances include ohmic resistance $\mathrm{R}_{0}$, electrochemical polarization resistance $R_{p a}$, and The equivalent capacitances which is electrochemical polarization capacitance $\mathrm{C}_{\mathrm{pa}}$ is used to describe the transient response during the 
charging/discharging transits. $\mathrm{i}_{\mathrm{L}}$ and $\mathrm{U}_{\mathrm{L}}$ are the charging/discharging current and the terminal voltage, respectively.

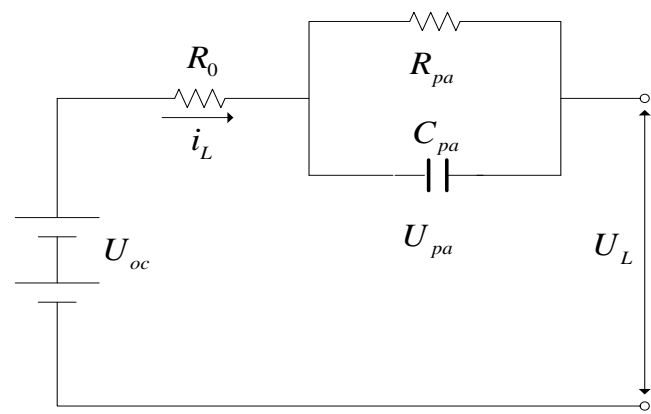

Fig.1 RC equivalent circuit

As shown in Fig. 1, the equation of RC equivalent circuit can be expressed as follows:

$$
\begin{aligned}
\dot{U}_{p a} & =-\frac{U_{p a}}{R_{p a} C_{p a}}+\frac{i_{L}}{C_{p a}} \\
\boldsymbol{U}_{L} & =\boldsymbol{U}_{o c}-\boldsymbol{U}_{p a}-i_{L} \boldsymbol{R}_{\mathrm{O}}
\end{aligned}
$$

Discrete state equation and observation equation discrete output:

$$
\left[\begin{array}{c}
S_{k+1} \\
U_{k+1}
\end{array}\right]=\left[\begin{array}{cc}
1 & 0 \\
0 & \exp \left(-\Delta t / \tau_{1}\right)
\end{array}\right]\left[\begin{array}{l}
S_{k} \\
U_{k}
\end{array}\right]+\left[\begin{array}{c}
-\Delta t \eta / C \\
R_{p a}\left(1-\exp \left(-\Delta t / \tau_{1}\right)\right)
\end{array}\right] i_{k}+\omega_{k}
$$

Where $\mathrm{C}$ is the battery nominal capacity, the unit is Ah; Coulomb factor $\eta$, Charging $\eta=1$, discharging $\eta<1 ; \tau_{1}=R_{p a} C_{p a}$ is time constant; $\omega_{k}, v_{k}$ are uncorrelated system noise.

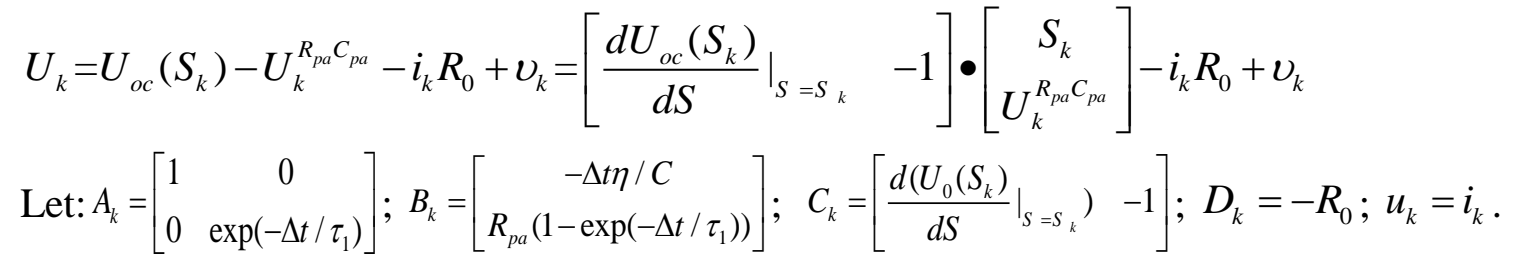

So: $x_{k+1}=f\left(x_{k}, u_{k}\right)+\omega_{k}=A_{k} x_{k-1}+B_{k} u_{k}+\omega_{k}$

$y_{k}=h\left(x_{k}, u_{k}\right)+v_{k}=C_{k} x_{k}+D_{k} u_{k}+v_{k}$

Traditional UKF algorithm is usually assumed process noise and measurement noise with mean zero, However, in practice, zero-mean noise conditions are not able to meet, so the first amendment to the nonlinear systems:

$$
\begin{aligned}
& x_{k+1}=f\left(x_{k}, u_{k}\right)+\omega_{k}+q_{k} \\
& y_{k}=h\left(x_{k}, u_{k}\right)+v_{k}+r_{k}
\end{aligned}
$$

Where, $E\left[\omega_{k}\right]=q_{k}, E\left[v_{k}\right]=r_{k}, \operatorname{cov}\left(\omega_{k}, \omega_{j}\right)=Q_{k} \delta_{k, j}, \operatorname{cov}\left(v_{k}, v_{j}\right)=R_{k} \delta_{k, j}, \operatorname{cov}\left(\omega_{k}, v_{j}\right)=0$.

\section{UT Transformation}

UT transformation is the core technology of UKF, UT ideological transformation is determined by a set of carefully chosen samples and gives samples corresponding weights to approximate a probability density function. A certain number of Sigma points were selected from the prior distribution according to a certain policy, the implementation of non-linear transformation for each Sigma point, so as to obtain the corresponding sampling point after conversion, Calculate posterior mean and variance using these samples weighting. The resulting samples by UT converted did not undergo linearized and higher order terms are not lost, so a higher accuracy than the EKF algorithm.

For any non-linear function $\mathrm{y}=\mathrm{g}(\mathrm{x})$, surrounding the $\mathrm{n}$ dimensional state variable $\mathrm{X}$ with the mean $\bar{x}$ and variance $p_{x}$, Choose $2 \mathrm{n}+1$ a Sigma Point $\chi_{i}$ and weights $\omega_{i}$ by the following process of UT transformation: 


$$
\begin{array}{ll}
\chi_{i}=\bar{x} & \mathrm{i}=0 \\
\chi_{i}=\bar{x}+\left(\sqrt{(n+\lambda) P_{x}}\right)_{i} & \mathrm{i}=1, \ldots, \mathrm{n} \\
\chi_{i}=\bar{x}-\left(\sqrt{(n+\lambda) P_{x}}\right)_{i-n} & \mathrm{i}=\mathrm{n}+1, \ldots, 2 \mathrm{n} \\
\omega_{0}^{m}=\omega_{0}^{c}=\lambda /(n+\lambda) & \mathrm{i}=0 \\
\omega_{i}^{m}=\omega_{i}^{c}=\lambda /(2(n+\lambda)) & \mathrm{i}=1, \ldots, 2 \mathrm{n}
\end{array}
$$

Where, $\left(\sqrt{(n+\lambda) P_{x}}\right)_{i}$ is $(n+\lambda) P_{x}$ square root of the $\mathrm{i}$ column or row. $\lambda$ is a scaling factor, used to characterize the sampling point range around the mean point, $\omega_{i}^{m}$ is the weight corresponding to the sample point. In the paper $\lambda=0.1, \mathrm{i}=2$.

Obtain samples by nonlinear transformation from $\chi_{i}$ :

$y_{i}=f\left(\chi_{i}\right)$

Then obtained approximate output samples weighted statistical characteristics:

$$
\begin{aligned}
& \hat{y}=\sum_{i}^{2 n} \omega_{i}^{m} y_{i} \\
& P_{y}=\sum_{i=0}^{2 n} \omega_{i}^{c}\left(y_{i}-\hat{y}\right)\left(y_{i}-\hat{y}\right)^{T}
\end{aligned}
$$

\section{General UKF Algorithm}

General UKF algorithm steps are as follows:

(1) Initialization

$$
\begin{aligned}
& \hat{x}_{0}=E\left(x_{0}\right) \\
& P_{0}=E\left[\left(x_{0}-\hat{x}_{0}\right)\left(x_{0}-\hat{x}_{0}\right)^{T}\right]
\end{aligned}
$$

(2) Sigma point calculation

$$
\chi_{i}=\left[\bar{x}, \bar{x}+\left(\sqrt{(n+\lambda) P_{x}}\right)_{i}, \bar{x}-\left(\sqrt{(n+\lambda) P_{x}}\right)_{i-n}\right]
$$

(3) Time Update

$$
\begin{aligned}
& \chi_{i, k / k-1}=f\left(\chi_{i, k-1}\right) \\
& \hat{x}_{k / k-1}=\sum_{i=0}^{2 n} \omega_{i}^{m} \chi_{i, k / k-1}+q_{k} \\
& P_{k, k-1}^{x}=\sum_{i=0}^{2 n} \omega_{i}^{c}\left[\chi_{i, k / k-1}-\hat{x}_{k / k-1}\right]\left[\chi_{i, k / k-1}-\hat{x}_{k / k-1}\right]^{T}+Q_{k} \\
& y_{i, k / k-1}=h\left(\chi_{i, k / k-1}\right) \\
& \hat{y}_{i, k / k-1}=\sum_{i=0}^{2 n} \omega_{i}^{m} y_{i, k / k-1}+r_{k}
\end{aligned}
$$

(4) Measurement Update

$$
\begin{aligned}
P_{y} & =\sum_{i=0}^{2 n} \omega_{i}^{c}\left(y_{i, k / k-1}-\hat{y}_{k / k-1}\right)\left(y_{i, k / k-1}-\hat{y}_{k / k-1}\right)^{T}+R_{k} \\
P_{x y} & =\sum_{i=0}^{2 n} \omega_{i}^{c}\left[\chi_{i, k / k-1}-\hat{x}_{k / k-1}\right]\left[y_{i, k / k-1}-\hat{y}_{k / k-1}\right]^{T} \\
K_{k} & =P_{x y} P_{y}^{-1}
\end{aligned}
$$




$$
\begin{aligned}
& \hat{x_{k}}=x_{k}+K_{k}\left[y_{k}-\hat{y}_{k / k-1}\right] \\
& P_{k}=P_{k / k-1}-K_{k} P_{y} K_{k}^{T}
\end{aligned}
$$

\section{AUKF algorithm}

Inaccurate mathematical models and statistical characteristics often caused noise filtering divergence, to solve these problems, adaptive filtering techniques are extensively studied, among them, Sage-Husa suboptimal unbiased maximum a posteriori (MAP) noise estimators, calculation is simple, clear principle, it is widely used [6].

Based on the maximum a posteriori estimation theory and measurement $y_{k}=h\left(x_{k}, u_{k}, v_{k}\right)$, and get the following suboptimal MAP noise statistics estimator:

$$
\begin{aligned}
& \hat{q}_{k}=\left(1-d_{k}\right) \hat{q}_{k-1}+d_{k}\left[\hat{x}_{k}-\sum_{i=0}^{2 n} \omega_{i}^{m} f\left(\chi_{i, k-1 / k-1}\right)_{i}\right] \\
& \hat{Q}_{k}=\left(1-d_{k}\right) \hat{Q}_{k-1}+d_{k}\left[K_{k} \tilde{y}_{k / k-1} \tilde{y}_{k / k-1}^{T} K_{k}^{T}+P_{k / k}-\sum_{i=0}^{2 n} \omega_{i}^{c}\left(\chi_{i, k / k-1}-\hat{x}_{k / k-1}\right)\left(\chi_{i, k / k-1}-\hat{x}_{k / k-1}\right)^{T}\right] \\
& \hat{r}_{k}=\left(1-d_{k}\right) \hat{r}_{k-1}+d_{k}\left[\hat{y}_{k}-\sum_{i=0}^{2 n} \omega_{i}^{m} h\left(\chi_{i, k-1 / k-1}\right)_{i}\right] \\
& \hat{R}_{k}=\left(1-d_{k}\right) \hat{R}_{k-1}+d_{k}\left[\tilde{y}_{k / k-1} \tilde{y}_{k / k-1}^{T}-\sum_{i=0}^{2 n} \omega_{i}^{c}\left(y_{i, k / k-1}-\hat{y}_{k / k-1}\right)\left(y_{i, k / k-1}-\hat{y}_{k / k-1}\right)^{T}\right] \\
& \tilde{y}_{k / k-1}=y_{k}-\hat{y}_{k / k-1}
\end{aligned}
$$

Where, $d_{k}=\frac{1-b}{1-b^{k}}, \mathrm{k}=1,2, \ldots, \mathrm{n} ; \mathrm{b}$ is the forgotten factor, $0<\mathrm{b}<1$.

\section{Model Verification}

In this paper, the results of parameter identification: Rpa Cpa=30, Rpa $=0.0028, \mathrm{R} 0=0.07$. To verify algorithm convergence and SOC estimation accuracy by matlab tools, simulation results shown in Figure 2 and Figure 3.

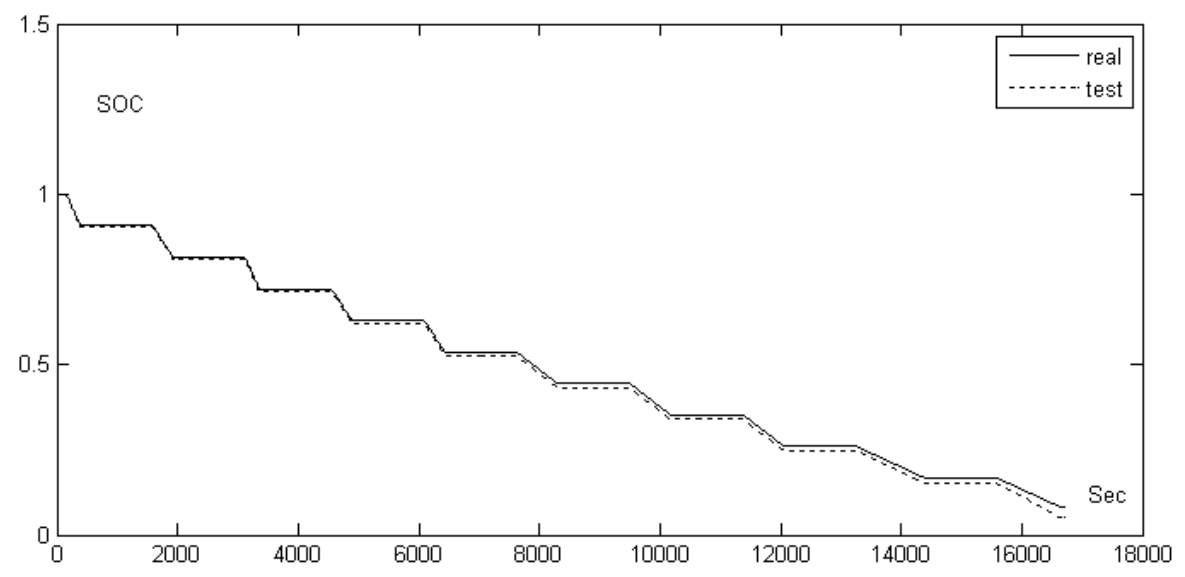

Fig. 2 simulation results of Power Lithium-ion Battery SOC I 


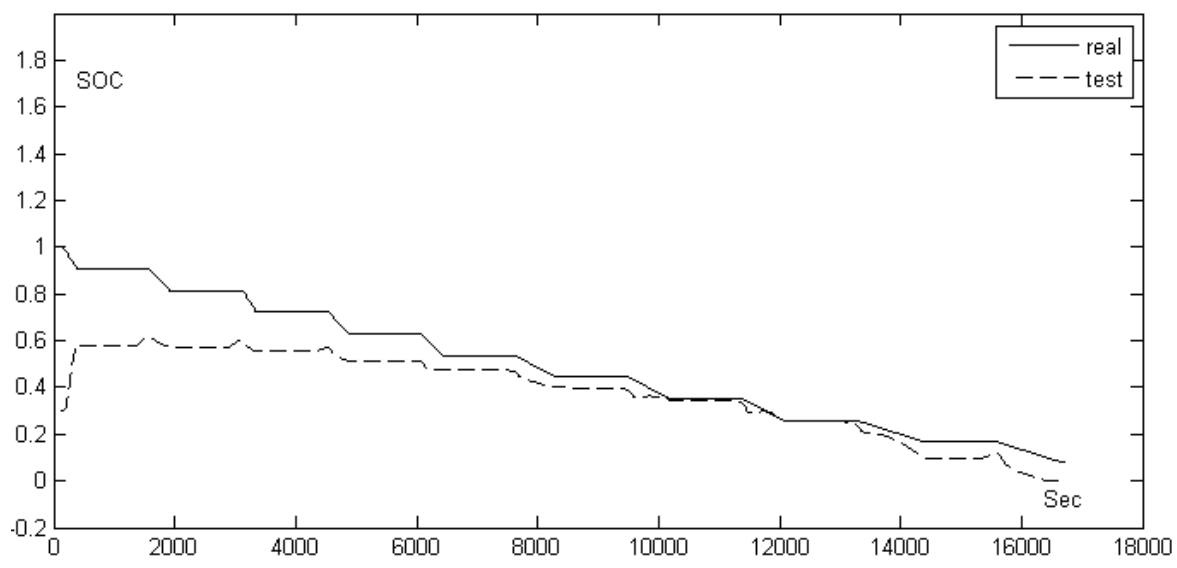

Fig. 3 simulation results of Power Lithium-ion Battery SOC II

In usual cases, SOC initial value is $1(100 \%)$, and then according to the plan to discharge, discharge curves of SOC, as shown in figure 2. Solid line for the actual test SOC value, dotted line to estimate based on AUKF algorithm, the figure shows that 2 curves consistency is very good, the error is very small;

In unusual cases, the initial SOC value of 0.3 (30\%), discharge data did not change; get the discharge curve of SOC, as shown in figure 3. Solid line for the actual test SOC value, dotted line based on AUKF algorithm estimates. The figure shows that when the initial value is not correct, AUKF algorithm can be adaptive adjustment, the convergence of come back soon, and relatively stable decreases with the measured values.

\section{Conclusion}

Through the simulation shows that power lithium battery SOC estimation method based on AUKF has some advanced and innovative. In the case of the initial value right, small errors; In the case of the initial value is wrong, is still very good convergence, can quickly adjust to the correct track.

\section{Acknowledgements}

This paper is supported by Shandong Provincial Natural Science Foundation, China (ZR2015FQ007 and ZR2014HM031).

\section{References}

[1] Cheng qing-quan, Sun feng-chun, Modern electric vehicle technology[m]. Beijing Institute of Technology Press. 2002: 238- 248.

[2] PILLER S, PERRIN M, JOSSENA. Methods for state of charge determination and their applications [J]. Journal of Power Sources, 2001, 96(1): 113-120.

[3] KIM K H, JEE GI, PARK CG. The stability analysis of the adaptive fading extended Kalman filter using the innovation covariance [J]. International Journal of Control, Automation, and Systems, 2009, 7(1): $49-56$.

[4] JWO DJ, WANG SH. Adaptive fuzzy strong tracking extended Kalman filtering for GPS navigation [J]. IEEE Sensors Journal, 2007, 7(5): 778 - 789.

[5] MAO Qun-hui, TENG Zhao-sheng, FANG Liang, Method of State of Charge Estimation Based on Unscented Kalman Filtering For Li-Ion Battery of Electric Vehicle [J]. Measurement \& Control Technology, 2010.29(3):89-91.

[6] SHI Yong, HAN Chong-Zhao. Adaptive UKF Method with Applications to Target Tracking [J]. ACTA AUTOMATICA SINICA, 2011, Vol.37, No.6: 755-759. 